\title{
Development and Extension of An Aggregated Scale Model: Part 1 - Background to ASMITA
}

\author{
Ian TOWNEND ${ }^{\mathrm{a}}$, Zheng Bing WANG ${ }^{\mathrm{b}, \mathrm{c}}$, Marcel STIVE ${ }^{\mathrm{b}}$ and Zeng ZHOU

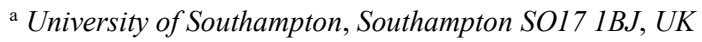 \\ ${ }^{\mathrm{b}}$ TU Delft, Delft 2600 AA, The Netherlands \\ ' Deltares, Delft 2600 MH, The Netherlands \\ d Jiangsu Key Laboratory of Coast Ocean Resources Development and Environment Security, \\ Hohai University, Nanjing 210098, China
}

(Received 22 April 2016; accepted 16 May 2016)

\begin{abstract}
Whilst much attention has been given to models that describe wave, tide and sediment transport processes in sufficient detail to determine the local changes in bed level over a relatively detailed representation of the bathymetry, far less attention has been given to models that consider the problem at a much larger scale (e.g. that of geomorphological elements such as a tidal flat and tidal channel). Such aggregated or lumped models tend not to represent the processes in detail but rather capture the behaviour at the scale of interest. One such model developed using the concept of an equilibrium concentration is the Aggregated Scale Morphological Interaction between Tidal basin and Adjacent coast (ASMITA). In this paper we provide some new insights into the concepts of equilibrium, and horizontal and vertical exchange that are key components of this modelling approach. In a companion paper, we summarise a range of developments that have been undertaken to extend the original model concept, to illustrate the flexibility and power of the conceptual framework. However, adding detail progressively moves the model in the direction of the more detailed process-based models and we give some consideration to the boundary between the two.

\section{Highlights}

- The concept of aggregating model scales is explored and the basis of the ASMITA model is outlined in detail;

- The relationship between dispersion as used in fast-scale process-based models and the horizontal exchange used in aggregated models is explored;

- The basis for formulating suitable equilibrium relationships is explained;

- Alternative ways to include advection and dispersion are examined.
\end{abstract}

Key words: estuary; tidal inlet; morphology; tides; waves; sediment transport

\section{Introduction}

The concept of an Aggregated Scale Morphological Interaction between Tidal basin and Adjacent coast (ASMITA) was first proposed by Stive et al. (1998) for the study of estuary and inlet response to sea-level rise. The idea behind ASMITA is that a tidal basin, or an estuary, can be sub-divided into a number of elements (Fig. 1). Each element is described in terms of its surface area, volume, and the horizontal and vertical rates of exchange. In addition, an equilibrium condition is associated with each type of element that equates to zero accommodation space. The model seeks to optimise these equilibrium conditions, subject to the initial conditions, any sources or sinks of sediment, and any imposed changes. For example, if under the initial conditions, the accommodation space is zero, there 
will be no net change in the transport of sediment. However, with an increasing mean sea level, the accommodation space becomes positive and sediment is imported (subject to availability); the opposite response occurs when sea level falls. The key concepts of the ASMITA model are:

- The estuary is schematized into a number of geomorphologic elements;

- The state of each element is described by its volume and surface area (water or sediment);

- Integrated parameters of hydrodynamics (tidal prism, or tidal range) are used;

- Empirical relationships define the morphological equilibrium for each element;

- Deviation from the morphological equilibrium causes a sediment demand (or supply); and

- A gradient in sediment demand (supply) drives sediment transport, and thereby morphological change.

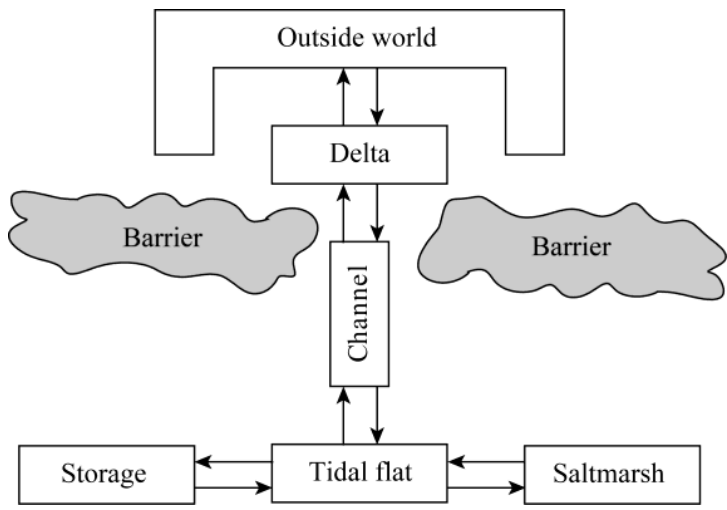

Fig. 1. Schematisation of an estuary or inlet into a number of elements as used in ASMITA.

The methodology was developed to look at the combined response of tidal delta, channel and tidal flats and has been applied to examine inlet response to sea level rise (van Goor et al., 2003), and human interferences (Kragtwijk et al., 2004), as well as changes in tidal range, such as the nodal tidal cycle (Jeuken et al., 2003; Wang and Townend, 2012). Kragwijk et al. (2004) also extended the application to multiple elements based on a matrix derivation of the governing equations.

These initial applications made use of fixed surface areas for the elements, and prescribed equilibrium conditions for the inlets derived from previous studies (Eysink, 1990; O'Brien, 1931; Renger and Partenscky, 1974). In these early applications, the parameters were used in conjunction with historical information to calibrate and validate the model. Following application to estuaries in the UK (Humber and Southampton Water), a more detailed appraisal of the model parameterisation was undertaken (Wang et al., 2008). This clarified the relationship of parameters such as the vertical and horizontal exchange within the aggregated model, ASMITA, and parameters that might be measured in the field. Different ways of schematising the geomorphology of an estuary using the three basic element types are linked and have also been explored against reported historical changes (Rossington et al., 2007). Direct comparisons with a more detailed process model (Delft 3D) have also provided further insight into the validity and limitations of the aggregated modelling approach (Dissanayake et al., 2011).

This paper provides a summary of these developments and explores the links with other 
formulations. In particular, we provide some new insights into the concepts of equilibrium, and horizontal and vertical exchange that are key components of this modelling approach. The application of the model is illustrated with a simple case study.

A number of additions have been added to the original formulation over the last decade. These include such things as river discharge, tidal pumping, littoral drift, saltmarsh elements, variable surface area and the influence of waves, and these are detailed more fully in a companion paper (Townend et al., 2016) referred to as Part 2.

\section{Outline of ASMITA Formulation}

The original model considered the mass balance within each element, based on horizontal exchanges in and out of the element and vertical exchanges to and from the bed. Two assumptions were then made:

(i) that there is an equilibrium state that can be prescribed in some way; and

(ii) that any deviation from the equilibrium in terms of sediment concentration, can be related to the flow velocity, and hence cross-sectional area, or volume (see discussion on equilibrium sediment concentration below).

Taking a single element model, such as an estuary channel, for simplicity, the first of these assumptions makes use of a relationship between the element volume and the tidal prism:

$$
V_{\mathrm{e}}=f(P),
$$

where $V_{\mathrm{e}}$ is the equilibrium volume of the channel and $P$ is the tidal prism (Eysink, 1990).

The second assumption leads to an equivalence between concentration and volume, and the local equilibrium concentration, $c_{\mathrm{e}}$, can be written in terms of actual volume, $V$, and equilibrium volume, $V_{\mathrm{e}}$ :

$$
c_{\mathrm{e}}=c_{\mathrm{E}}\left(\frac{V_{\mathrm{e}}}{V}\right)^{n},
$$

where $n$ is the concentration transport exponent and $c_{\mathrm{E}}$ is the equilibrium concentration for the system as a whole (usually taken as the open boundary value). The difference between the actual concentration and the local equilibrium value induces morphological change governed by the exchange between the water column and the bed:

$$
\frac{\mathrm{d} V}{\mathrm{~d} t}=\frac{1}{c_{\mathrm{b}}} w S\left(c_{\mathrm{e}}-c\right)
$$

where $w$ is the vertical exchange coefficient, $S$ is the surface area of the channel, $c$ is the actual concentration and $c_{\mathrm{b}}$ is the concentration of the bed (omitted in the original derivation). In addition, any vertical exchange must be balanced by the horizontal exchange (assuming that, on the morphological time scale, the change in the amount of sediment in suspension is negligible compared to the amount of deposition/erosion):

$$
w S\left(c_{\mathrm{e}}-c\right)=\delta\left(c-c_{\mathrm{E}}\right) .
$$

The parameter $\delta$ is the horizontal exchange coefficient and, for a single element, the r.h.s. of Eq. (4) represents the exchange with the external environment. 
Combining Eqs. (2)-(4), the morphological change in volume is given by:

$$
\frac{\mathrm{d} V}{\mathrm{~d} t}=\frac{1}{c_{\mathrm{b}}} \cdot \frac{w \delta c_{\mathrm{E}} S}{\delta+w S}\left[\left(\frac{V_{\mathrm{e}}(t)}{V(t)}\right)^{n}-1\right] .
$$

This is a change relative to the initial volume and so does not include changes in water levels and tidal range. Therefore, the morphological change within the existing volume can be thought of as the volume change relative to a fixed water surface, $V_{\mathrm{f}}$. Including changes in water surface and morphological changes gives the total volume change, or volume relative to a moving water surface, $V_{\mathrm{m}}$. Adopting a simple linear relationship for the equilibrium volume of the form $V_{\mathrm{e}}=\alpha P$, where $\alpha$ is an empirical coefficient, these three volumes are given by:

$$
\begin{aligned}
& V_{\mathrm{e}}(t)=\alpha \cdot P(t)=\alpha \cdot\left(P_{0}+\Delta P\right) ; \\
& V_{\mathrm{f}}(t)=V_{\mathrm{f} 0}+\int \frac{\mathrm{d} V}{\mathrm{~d} t} \mathrm{~d} t \\
& V_{\mathrm{m}}(t)=V_{\mathrm{m} 0}+\int \frac{\mathrm{d} V}{\mathrm{~d} t} \mathrm{~d} t+S \int \frac{\mathrm{d} \zeta}{\mathrm{d} t} \mathrm{~d} t,
\end{aligned}
$$

where $\Delta P$ is the change in prism, $\zeta$ is a vertical change in the reference water level (this may include changes in mean water level and tidal range depending on the reference surface for the particular element type), and the subscript " 0 " refers to the initial condition. In Eq. (6) $\mathrm{d} V / \mathrm{d} t$ is obtained from Eq. (5) and this can be implemented numerically using a simple forward stepping scheme subject to a suitable choice of time step (see Appendix). Alternative definitions of equilibrium are readily considered. For example, the above expression could be replaced by $V_{\mathrm{e}}=\alpha P^{\beta}$, where $\beta$ is a coefficient to define the non-linear variation of volume as a function of tidal prism.

The above equations are for a single element but can readily be extended to multiple elements. The original development considered the tidal delta, tidal flats and a channel as components of the tidal inlet (Kragtwijk et al., 2004; van Goor et al., 2003). The variation in these volumes depends on the transport of sediment in and out of the elements and any changes to the water volume itself. The latter may be due to sea level rise, subsidence of the bed, or any form of progressive change in the basin volume. Hence, over the long-term (time scales much longer than a tidal cycle), the rate of change of the element volume depends on the residual flux, and any change in reference water surface (e.g. due to sea level rise or tidal range variations):

$$
\frac{\mathrm{d} V_{i}}{\mathrm{~d} t}=\frac{1}{c_{\mathrm{b} i}} \sum_{j} J_{i, j}+S_{i} \frac{\mathrm{d} \zeta}{\mathrm{d} t},
$$

where $J$ is the sediment flux between elements $i$ and $j$. If the residual sediment flux between two elements is assumed to have advective and dispersive components:

$$
J_{i, j}=q_{i, j} \cdot\left(\frac{c_{i}+c_{j}}{2}\right)+D_{i, j} A_{i, j} \frac{\partial c}{\partial x}=q_{i, j} \cdot\left(\frac{c_{i}+c_{j}}{2}\right)+\delta_{i, j} \cdot\left(c_{i}-c_{j}\right),
$$

where $q\left(\mathrm{~m}^{3} \mathrm{~s}^{-1}\right)$ is the residual discharge rate, $D\left(\mathrm{~m}^{2} \mathrm{~s}^{-1}\right)$ is the inter-tidal dispersion coefficient between the two elements (i.e. tidal integrated), $A\left(\mathrm{~m}^{2}\right)$ is the vertical area of the interface between the two elements. 
In Eq. (8) the subscripts $i$ and $j$ refer to the elements that the transport is from and to respectively. Some additions, outlined in Part 2, extend the model to include storage areas and saltmarsh elements (Fig. 1). It is also possible to subdivide elements such as the channel into multiple elements. The equations for such cases are best written in matrix form, as proposed by Kragtwijk et al. (2004), and are detailed in the Appendix.

It should be noted that the equations presented are for long-term fluxes and so there is not a direct equivalence between for example the vertical flux and the settling velocity commonly used in process models. The issue of parameter selection was explored by Wang et al. (2008) and a typical calibration procedure is as follows:

- use the exponent in a suitable sediment transport formula to define the transport coefficient, $n$. Typically this would have a value between 2 and 5 depending on the type of sediment;

- $\quad$ base the vertical exchange, $w$, on the settling velocity;

- the dispersion coefficient $D$ should be proportional to $u^{2} H / w$ in which $u$ is the scale of the tidal flow velocity, $H$ is the hydraulic water depth and $w$ is the rate of vertical exchange. The horizontal exchange coefficient, $\delta\left(\mathrm{m}^{3} \mathrm{~s}^{-1}\right)$, is then obtained from the dispersion coefficient as follows:

$$
D=\varepsilon \frac{u^{2} H}{w} \quad \text { and } \quad \delta_{i, j}=\frac{D_{i, j} A_{i, j}}{\Delta x},
$$

- where $\Delta x$ is a representative distance between the two elements. The coefficient of proportionality, $\varepsilon$, should have a value of around 0.1 , but is used as a calibration parameter to ensure the correct level of mixing.

- The choice of global equilibrium concentration, $c_{\mathrm{E}}$, is guided by the background concentration in the marine environment (especially if tidal pumping is included as explained in Part 2) or the average concentration within the estuary, if the system is believed to be close to equilibrium. The final choice may be adjusted to ensure that the combined influence of the transport coefficient, $n$, and equilibrium concentration, $c_{\mathrm{E}}$, give the correct morphological time scale.

\subsection{Equilibrium Sediment Transport}

A very similar approach has been developed in terms of the (tidally averaged) equilibrium sediment concentration. Sediments are moved in and out during the flood and ebb tide. Over short time scales (seconds-hours), this transport is largely advective in character. However, over longer time scales of one or more tides (days-years), the redistribution of sediment is characterised by the tidally averaged dispersion. Following this line of reasoning, Di Silvio et al. (2010) proposed "a tidally averaged equilibrium concentration as the local transport concentration value which, with the prevailing local shear stresses, does not produce net erosion or deposition". This definition formalised earlier proposals for a model that considered tidally averaged flow and concentration to determine erosion and accretion within a tidal basin based on a suitably defined equilibrium concentration (Dal Monte and Di Silvio, 2004; Di Silvio, 1989). Rather than using aggregated "elements", sediment transport was computed on a Cartesian grid, such that: 


$$
\frac{\partial J_{x}}{\partial x}+\frac{\partial J_{y}}{\partial y}=F
$$

where $J_{x}$ and $J_{y}$ are the sediment fluxes in the $x$ and $y$ directions, and $F$ is the net erosion or deposition. As outlined above in Eq. (8), the fluxes were then computed in terms of the advective and dispersive contributions. The erosion/deposition rate made use of the depth integrated model proposed by Gallappatti and Vreugdenhil (1985), integrated over a tidal cycle:

$$
F=w\left(c_{\mathrm{E}}-c\right),
$$

where $w$ is the rate of vertical exchange with the bed, $c$ is the concentration and $c_{\mathrm{E}}$ is the equilibrium concentration. These two equations then provided what was needed to solve the equation for the rate of bed level change, given by:

$$
\frac{\mathrm{d} h}{\mathrm{~d} t}=\frac{w}{c_{\mathrm{b}}}\left(c_{\mathrm{E}}-c\right)+\alpha,
$$

where $c_{\mathrm{b}}$ is the concentration of the bed and $\alpha$ accounts for other changes such as sea level rise or land subsidence (Di Silvio, 1989). The concentration here is the instantaneous concentration, $c(t)$, averaged over a sufficiently long time. By noting the relationship between discharge and velocity and using a suitable sediment transport equation, where $q_{\mathrm{s}}$ is proportional to $u^{n}$ ( $u$ is the velocity), the depth averaged instantaneous concentration is given by:

$$
c=\frac{q_{\mathrm{s}}}{q} \propto \frac{u^{n}}{q} \propto \frac{u^{n-1}}{h^{n}} .
$$

By averaging over a suitable interval, the long-term averaged concentration is obtained. Eq. (13) is used to define relationships for tides, internal and external waves, and river inputs, which are summed to give a total equilibrium concentration (Di Silvio et al., 2010). For a system close to equilibrium, this equates to the global equilibrium concentration used in the ASMITA formulation. It therefore follows that the calibration process used to set up the Di Silvio model would be one way of determining the value of the global equilibrium concentration, $c_{\mathrm{E}}$.

Within the element itself, we assume that of the material that moves in and out on every tide, some will fall to the bed, or be eroded from the bed, depending on whether the element is above or below its equilibrium condition. Over the long-term time scale, this can also be considered as a dispersion process. For a suspended sediment load and given flow conditions, the equilibrium bed level as a function of the flow field, bed strength, and dispersion coefficient, depends on the concentration velocity, defined as (Seymour, 2004, pers.com.):

$$
\phi=\frac{\tau_{\mathrm{o}}-\tau_{\mathrm{cr}}}{D},
$$

where $D$ is again the dispersion coefficient, $\tau_{\mathrm{o}}$ is the bed shear stress and $\tau_{\mathrm{cr}}$ is critical bed shear stress for erosion. When the concentration velocity tends to zero, there is no net erosion or deposition and the concentration of suspended particles is in equilibrium. At equilibrium within a particular element, the concentration is proportional to the shear stress, which is a function of velocity squared. Now as the velocity is inversely proportional to the volume of the element, the equilibrium concentration for the 
element can be written in terms of the equilibrium concentration for the system as a whole, $c_{\mathrm{E}}$. We also make the assumption that the actual transport in an element is approximately equal to the potential transport, so that $c_{i}=c_{i \mathrm{e}}$ and can be related to $c_{\mathrm{E}}$ as follows:

$$
c \propto \tau \propto u^{2} \propto 1 / V^{2} \quad \text { so that } \frac{c_{i \mathrm{e}}}{c_{\mathrm{E}}}=\left(\frac{V_{i \mathrm{e}}}{V_{i}}\right)^{2} .
$$

When in equilibrium, the concentration in all elements will tend to $c_{\mathrm{E}}$. This overall, or global, equilibrium concentration is usually defined at the open sea boundary, if this can be considered in equilibrium. However, it does not follow that this should be the background concentration found in the open sea. Estuaries are known to have significantly higher suspended sediment concentrations moving back and forth on every tide than those typically found on the open coast. This is, in part, a reflection of the concentration velocity to achieve equilibrium but also influences such as tidal asymmetry, which is introduced as an addition in Part 2 using the concept of tidal pumping. It may therefore be more appropriate to consider the long-term average concentration of the estuary as a whole as the representative equilibrium concentration, $c_{\mathrm{E}}$. This is consistent with the approach of Di Silvio et al. (2010), who used various forms of Eq. (13) to sum the contributions to the equilibrium concentration within the tidal basin.

\subsection{Aggregation of the Sediment Concentration Equation}

In this section we illustrate how the mass-balance equations in ASMITA can be derived from the three-dimensional (3D) convection-diffusion equation for sediment concentration. The derivation serves to: (1) clarify the equivalence and differences between ASMITA and a process-based morphodynamic model (e.g. Delft3D); and (2) identify the physical nature of the (intertidal) dispersion.

The 3D convection-diffusion equation for sediment concentration reads:

$$
\frac{\partial c}{\partial t}+\frac{\partial(u c)}{\partial x}+\frac{\partial(v c)}{\partial y}+\frac{\partial(w c)}{\partial z}-\frac{\partial}{\partial x}\left(\varepsilon_{x} \frac{\partial c}{\partial x}\right)-\frac{\partial}{\partial y}\left(\varepsilon_{y} \frac{\partial c}{\partial y}\right)=w_{\mathrm{s}} \frac{\partial c}{\partial z}+\frac{\partial}{\partial z}\left(\varepsilon_{z} \frac{\partial c}{\partial z}\right)
$$

Herein $c=$ sediment concentration; $t=$ time; $u, v=$ horizontal flow velocity components;

$x, y=$ horizontal coordinates; $w=$ vertical flow velocity; $z=$ vertical coordinate;

$\varepsilon_{x}, \varepsilon_{y}, \varepsilon_{z}=$ turbulent diffusion coefficients; $w_{\mathrm{s}}=$ settling velocity.

To solve this equation a bed-boundary condition is needed. At the boundary near the bed the sediment concentration (which equates to the downwards flux when multiplied by the settling velocity) or the sediment concentration gradient (which equates to the upwards flux when multiplied by the vertical diffusion coefficient) can be prescribed. Their values need to be calculated using a sediment transport formula (in the case of sand) or a formulation for the erosion rate (in the case of mud).

Eq. (16) can first be integrated in the vertical direction to obtain the depth-averaged advectiondiffusion equation:

$$
\frac{\partial(h \bar{c})}{\partial t}+\frac{\partial\left(\alpha_{x} \bar{u} h \bar{c}\right)}{\partial x}+\frac{\partial\left(\alpha_{y} \bar{v} h \bar{c}\right)}{\partial y}-\frac{\partial}{\partial x}\left(D_{x} h \frac{\partial \bar{c}}{\partial x}\right)-\frac{\partial}{\partial y}\left(D_{y} h \frac{\partial \bar{c}}{\partial y}\right)=E-D_{\mathrm{b}}
$$

with the over-bar representing depth-averaging of the corresponding variable, and $h=$ water depth; $\alpha_{x}$, 
$\alpha_{y}=$ coefficients counting for the effects of the shapes of the vertical distribution of flow velocity and sediment concentration; $D_{x}, D_{y}=$ dispersion coefficients; $E=$ erosion at the bottom; $D_{\mathrm{b}}=$ deposition to the bottom.

This is the first level of aggregation as used by many process-based models. Note that the diffusion coefficients in the horizontal direction become dispersion coefficients as they also represent the mixing due to the non-uniform vertical distributions of the flow velocity and the sediment concentration.

For non-cohesive sediment (sand) the sediment exchange between the bottom and the water column can be derived using an asymptotic solution of the convection-diffusion Eq. (16) (Gallappatti and Vreugdenhil, 1985; Wang, 1992). For cohesive sediment (mud) these terms can be calculated using e.g. the Krone-Partheniade formulation. In general, such formulations have the form

$$
\begin{aligned}
& E=f(\tau, \ldots) ; \\
& D_{\mathrm{b}}=\gamma w_{\mathrm{s}} \bar{c} .
\end{aligned}
$$

It is important to mention that the erosion, $E$, is independent of the sediment concentration, whereas the deposition, $D_{\mathrm{b}}$, is proportional to the sediment concentration. The coefficient, $\gamma$, is in general a function of $w_{\mathrm{s}} / u^{*}$ in which $u^{*}$ is the bed shear velocity.

Eq. (17) can be written as:

$$
\frac{\partial(h \bar{c})}{\partial t}+\frac{\partial s_{x}}{\partial x}+\frac{\partial s_{y}}{\partial y}=E-D_{\mathrm{b}},
$$

in which $s_{x}$ and $s_{y}$ represent the suspended sediment transport rate in the $x$ - and $y$-direction. This equation can further be aggregated by integrating over a part, or the whole, of the estuary (a morphological element). Using Green's theorem, the integration yields:

$$
\frac{\partial(V C)}{\partial t}=\sum_{i} S_{i}+\text { Source }- \text { Sink, }
$$

herein $V$ is the volume of the water body of the area and $S_{i}$ denotes sediment transport at open boundary (positive=import).

This equation can also be directly derived by considering the mass-balance of sediment in the whole water body. The Source and Sink terms represent the integrals, over some area, A, of respectively $E$ and $D_{\mathrm{b}}$.

$$
\begin{aligned}
& \text { Source }=\iint_{A} E \mathrm{~d} x \mathrm{~d} y+\text { External Sources; } \\
& \text { Sink }=\iint_{A} D \mathrm{~d} x \mathrm{~d} y .
\end{aligned}
$$

Eqs. (16), (17), (20) and (21) can also be aggregated in time, e.g. over a tidal period or a much longer time. As an example, integration of Eq. (17) over a tidal period yields:

$$
\frac{\partial\left(u_{\mathrm{r}} h \bar{c}\right)}{\partial x}+\frac{\partial\left(v_{\mathrm{r}} h \bar{c}\right)}{\partial y}-\frac{\partial}{\partial x}\left(D_{x} h \frac{\partial \bar{c}}{\partial x}\right)-\frac{\partial}{\partial y}\left(D_{y} h \frac{\partial \bar{c}}{\partial y}\right)=E-D_{\mathrm{b}} .
$$

The first term representing the change of sediment storage in the water column is neglected as it becomes much less important on longer time-scale than the terms on the right hand side representing 
the exchange with the bottom. All the other terms remain basically the same, but the parameters and variables now represent the tidally-averaged values. The residual flow velocities $\left(u_{\mathrm{r}}, v_{\mathrm{r}}\right)$ causes advection and the tidal flow now becomes the major mixing agent for the dispersion represented by the coefficients $D_{x}$ and $D_{y}$, as elaborated by Wang et al. (2008).

Aggregation in time of Eq. (21) yields the equation used by ASMITA.

$$
\sum_{i} S_{i}+\text { Source }- \text { Sink }=0 .
$$

Due to the aggregations the Source and Sink terms can no longer be calculated using independent formulae for sediment transport and bed erosion, as the required detailed information on flow strength is no longer available. However, the aggregation over morphological elements also makes it possible to use empirical relations to calculate the morphological equilibrium, which are used to calculate the Source and Sink terms (see previous sections).

The ASMITA model is thus based on the same principles as a process-based model (e.g. Delft3D) in the case that the suspended sediment transport is dominant. Both models try to represent the same physical processes, but at different levels of aggregation. The difference between the two models is in the formulations for the exchange between the bottom sediment and the water column. In Delft3D this is arranged via the bottom boundary condition in $3 \mathrm{D}$ mode or a formulation derived from an asymptotic solution of the (3D) advection-diffusion equation (Galappatti and Vreugdenhil, 1985; Wang, 1992) in 2DH mode. In both cases, the local equilibrium concentration for sediment needs to be calculated. This requires a sediment transport formula that relates the transport capacity to the strength of the flow and the properties of sediment. A sediment transport formula is often derived by considering the relevant physical processes, but it always contains parameter (s) to be calibrated with observations from the field and/or laboratory. In that sense, a sediment transport formula is thus empirical. One must also take into account the uncertainties associated with the application of such a formula. This is shown by the fact that there are not one but many sediment transport formulas available. An indication of the uncertainty is given by van Rijn (1984) who suggested that sediment transport estimates and measurements can differ by a factor of 2. In ASMITA, a single formulation is used for the exchange between the bottom and the water column. This is based on relationships between the morphological equilibrium of the elements and aggregate hydrodynamic parameters, reflecting the aggregation in time and space. These relationships are, like the sediment transport formula, based on physical considerations (to determine relevant hydrodynamic parameters and morphological relationships) and observations from the field (to calibrate the parameters in the relationship). The local equilibrium concentration is related to the ratio between the equilibrium volume and the actual volume. The empirical relationships for the morphological equilibrium also contain uncertainties, just like the empirical aspects of a sediment transport formula in Delft3D.

\subsection{Dispersion}

Dispersion is used here to describe the horizontal exchange (between nodes or elements) and can be derived from 3D convection-diffusion equation, as just outlined. Since this is a process which seeks to reduce or smooth gradients in concentration, at the time-scale of interest (decades), it might also be referred to as diffusion. Here we follow Dronkers and use the term dispersion for model scale 
processes and diffusion for processes that take place at sub-model scale (Dronkers, 2005). The dispersion occurs in the definition of the horizontal fluxes in zero-dimensional form in Eq. (8) and in two-dimensional form in the equivalent expansion of the fluxes used in Eq. (9). For the latter Di Silvio et al. (2010) followed the approach of Dronkers (1978) and used a tensor to describe the twodimensional tidal dispersion as:

$$
\boldsymbol{D}=\left(\begin{array}{ll}
D_{x x} & D_{x y} \\
D_{y x} & D_{y y}
\end{array}\right)=k_{\mathrm{e}}\left(\begin{array}{cc}
u^{2} & u v \\
v u & v^{2}
\end{array}\right),
$$

where $k_{\mathrm{e}}$ is coefficient and $u$ and $v$ are suitably averaged velocity fields. Recognising that $k_{\mathrm{e}}$ is poorly defined and as a result is used as a poorly constrained calibration parameter, Zhou (pers.comm., 2012) examined the oscillatory shear stress, $K_{0}$, based on the work of Lewis (1997), who proposed:

$$
K_{\mathrm{o}}=\frac{u_{\mathrm{s}}^{2} h^{2}}{A_{\mathrm{L}} K_{z}},
$$

where $u_{\mathrm{s}}$ is the surface velocity, $K_{z}$ is the vertical shear dispersion coefficient (typically $0.0001-0.001$ $\mathrm{m}^{2} / \mathrm{s}$, depending on the vertical mixing strength), $h$ is water depth, $A_{\mathrm{L}}$ is a scaling parameter that is a function of the vertical velocity profile, $A_{\mathrm{L}}=f(\beta)$, described by:

$$
u_{z}=u_{\mathrm{s}}\left(1-\frac{z}{h}\right)^{\beta} \quad \text { for } 0 \square \quad \square .
$$

Hence, ignoring the molecular diffusion and steady state shear contribution, the effective dispersion coefficient can be written as:

$$
K_{\mathrm{o}}=\frac{(\beta+1)^{2} h}{c_{v} A_{\mathrm{L}} \sqrt{u^{2}+v^{2}}}\left(\begin{array}{ll}
u^{2} & u v \\
v u & v^{2}
\end{array}\right) .
$$

where $c_{v}$ is a constant ( 0.002). Comparing Eqs. (26) and (29), this clearly provides a more physically based definition of $k_{\mathrm{e}}$. For a typical tidal estuary with a velocity of around $1 \mathrm{~m} / \mathrm{s}$, a water depth of 10 $\mathrm{m}$, and assuming a linear vertical profile ( $\beta=1)$, Eq. (29) suggests a value of $A_{\mathrm{L}}=120$ and dispersion coefficient of $150 \mathrm{~m}^{2} / \mathrm{s}$.

An alternative form of the ASMITA concept, made use of a 1-D flow model and so considered cross-sections along the channel rather than reaches (Wang et al., 1998). The linearised form of the equations for morphological development from this model was subsequently elaborated (Wang and Townend, 2012) and used to examine the form of the intertidal dispersion coefficient (Wang et al., 2008). As already noted, Eq. (9), this suggested that the inter-tidal dispersion coefficient $D$ should be proportional to $u^{2} H / w$, which is consistent with the more detailed 2-D form presented here.

\section{Additional Considerations}

\subsection{Alternative Equilibrium Conditions}

\subsubsection{Empirical Prism Relationships}

The most often used form of equilibrium within ASMITA makes use of the O'Brien-Jarrett relationship (Jarrett, 1976; O'Brien, 1931). This relates cross-sectional area to tidal prism but the 
relationship with tidal prism has been shown to be equally applicable to channel and intertidal volumes (Eysink, 1991; Townend, 2005) and leads to an expression of the form: $V_{\mathrm{e}}=\alpha P^{\beta}$. For the tidal inlets that make up the Wadden Sea (Netherlands, Germany, Denmark), the channel volumes have been shown to follow an exponent of $\beta=1.5$ (Eysink and Biegel, 1992). From the UK database, which includes 66 estuaries of different types and size, this usually provides a good description for the volume at mean tide level but a statistically slightly poorer description of the channel and flats as separate elements (see Table 1). Similar relationships for the surface area are improved by scaling the tidal prism using the tidal range (Table 1). As can be seen from the goodness-of-fit values $\left(R^{2}\right)$ in Table 1, the addition of an exponent does not necessarily improve the quality of the fit. Hence using a linear relationship is a plausible option for estuaries but not necessarily for semi-enclosed basins. The single fit parameter, $\alpha$, is then simply the ratio of the volume to tidal prism. By assuming that an estuary, in its present state, is close to equilibrium, this coefficient can then be readily calculated. This is one of the simplest ways to set up the equilibrium condition in the ASMITA model.

Table 1 Tidal prism relationships based on the estuaries in the UK estuary database. Surface area relationships are based on the tidal prism divided by the tidal range.

\begin{tabular}{cccccccc}
\hline & & \multicolumn{2}{c}{ Linear } & \multicolumn{3}{c}{ Power law } \\
\cline { 3 - 7 } & & $\alpha$ & $R^{2}$ & $\alpha$ & $\beta$ & $R^{2}$ \\
\hline \multirow{3}{*}{ Volume } & MTL & 0.843 & 0.98 & 0.096 & 1.113 & 0.92 \\
& Channel & 0.418 & 0.93 & 0.005 & 1.230 & 0.80 \\
& Flat & 0.163 & 0.74 & 0.080 & 1.081 & 0.61 \\
\hline \multirow{3}{*}{ Surface area } & MTL & 1.08 & 0.99 & 0.377 & 1.049 & 0.96 \\
& Channel & 0.78 & 0.97 & 0.047 & 1.152 & 0.93 \\
& Flat & 0.95 & 0.77 & 31.0 & 0.810 & 0.83 \\
\hline
\end{tabular}

\subsubsection{Idealised Cross-Section As A Function of Tidal Prism}

An alternative approach is to use the equilibrium hypsometry relationships for channels (Cao and Knight, 1997) and tidal flats (Friedrichs and Aubrey, 1996) to define the form of the estuary crosssection (see Fig. 2). The equations to describe this (tidal flow only) form have been used to explore estuary form (Townend, 2010). However, integrating this cross-section over the respective intervals provides cross-sectional areas of the intertidal and channel, respectively. This leads to the following simple expressions for surface areas, $S$, and volumes, $V$, of the channel (lw) and intertidal flats (fl), where $n_{\mathrm{bk}}$ has a value of 1 or 2 depending on whether a half or full cross-section is being considered:

$$
\begin{aligned}
& S_{\mathrm{lw}}=\frac{P}{2 a}-n_{\mathrm{bk}} \cdot a \cdot m_{\mathrm{eqS}} ; \quad V_{\mathrm{lw}}=\frac{\mu}{n_{\mathrm{bk}}(n+1)} \cdot \frac{S_{\mathrm{lw}}{ }^{2}}{L_{\mathrm{e}}} ; \\
& S_{\mathrm{fl}}=\left(1+\frac{\pi}{2}\right) \cdot n_{\mathrm{bk}} \cdot a \cdot m_{\mathrm{eqS}} ; \quad V_{\mathrm{fl}}=\frac{1+\pi}{2} n_{\mathrm{bk}} \cdot a^{2} \cdot m_{\mathrm{eqs}} .
\end{aligned}
$$

In the above, $P$ is the tidal prism; $n$ determines the shape of the channel (the larger the value, the closer the form is to a rectangular channel) and is parabolic with a value of $n=2$; the equilibrium slope can be calculated from: $m_{\mathrm{eqs}}=\frac{W_{\mathrm{o}}-W_{\mathrm{lw}}}{n_{\mathrm{bk}} a} \cdot L_{\mathrm{e}}$, where the widths, $W_{\mathrm{o}}$ and $W_{\mathrm{lw}}$, are as defined in Fig. $2, L_{\mathrm{e}}$ is the estuary length and $a$ is the tidal amplitude. If the slope, $m_{\mathrm{eqs}}$ and shape coefficient, $n$, are treated 
as being constant, Eq. (30) can be used to derive updated equilibrium volumes and areas for the channels and flats given a new value of the tidal prism. Comparisons for a number of UK estuaries suggest that these equations provide a reasonable estimates of the gross properties. It also follows that the more complete description of estuary shape based on external parameters (rather than the tidal prism) and including influences such as internal wind-waves and saltmarsh (Townend, 2012) can also be used as a basis for prescribing equilibrium.

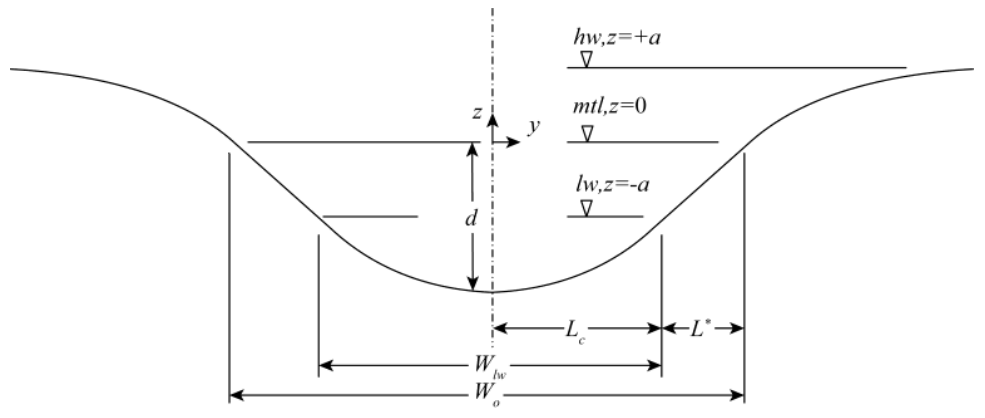

Fig. 2. Definition sketch for equilibrium form of channel and intertidal.

\subsubsection{Hypsometry As A Function of Tidal Prism}

The simplest form of hypsometry description is proposed by Wang et al. (2002). This is based on an empirical power law expression of the following form:

$$
V(z)=C \cdot(d+z)^{\alpha},
$$

where $V$ is the volume of the reach or estuary, $C$ and $\alpha$ are constants, $d$ is the depth of the section below the mean tide level datum, and $z$ is the elevation relative to the datum (positive above the datum). This can be written as:

$$
\frac{V(z)}{V_{\mathrm{o}}}=\left(1+\frac{z}{d}\right)^{\alpha},
$$

where $V_{\mathrm{o}}=C \cdot d^{\alpha}$ is the volume below the mean tide level datum. The derivative of equation is surface area of the reach or estuary, i.e:

$$
S(z)=\alpha C \cdot(d+z)^{\alpha-1},
$$

where $\alpha C \cdot d^{\alpha-1}$ is the surface area at the mean tide level datum, $S_{0}$, from which it follows that the average depth, or hydraulic depth, at any elevation is given by:

$$
H(z)=\frac{V(z)}{S(z)}=\frac{d+z}{\alpha} .
$$

So that $V_{\mathrm{o}} / S_{\mathrm{o}}=H_{\mathrm{o}}=d / \alpha$. These relationships have been shown to provide a good description of the hypsometry, as illustrated in Fig. 3. Making use of approximate estimates of the tidal prism using (i) the volume between high and low water slack water; and (ii) the tidal excursion through the area at the mouth (Savenije, 2005), one can derive relationships for the volume and surface area at mean tide level:

$$
S_{o}=\frac{P\left(1-\delta \cdot L_{w}\right)}{2 a \cdot \cos (\varepsilon)} \text { and } V_{o}=\frac{\omega P L_{A}}{2 u}
$$


where $\varepsilon$ is the phase lag between tidal elevation and tidal velocity; $\omega$ is the angular frequency of the tide $\left(2 \pi / T_{\mathrm{p}}\right), T_{\mathrm{p}}$ being the tidal period; $a$ is the tidal amplitude, $u$ is the tidal velocity amplitude, $\delta$ is the along-channel damping of tidal amplitude and $L_{A}$ and $L_{w}$ are the rates of area and width convergence. For UK estuaries, these both provide reasonable estimates, even without detailed knowledge of the phase lag and rates of convergence. The surface area fit is given in Table 1, based on $S_{\mathrm{p}} \alpha P /(2 a)$, the fit coefficient accounting for $\left(1-\delta \cdot L_{w}\right) / \cos \varepsilon$. The fit for volumes based on Eq. (35) is also quite good with $R^{2}=0.96$, although this still has quite a lot of scatter, probably reflecting the quality of the estimates of convergence and velocity amplitude.

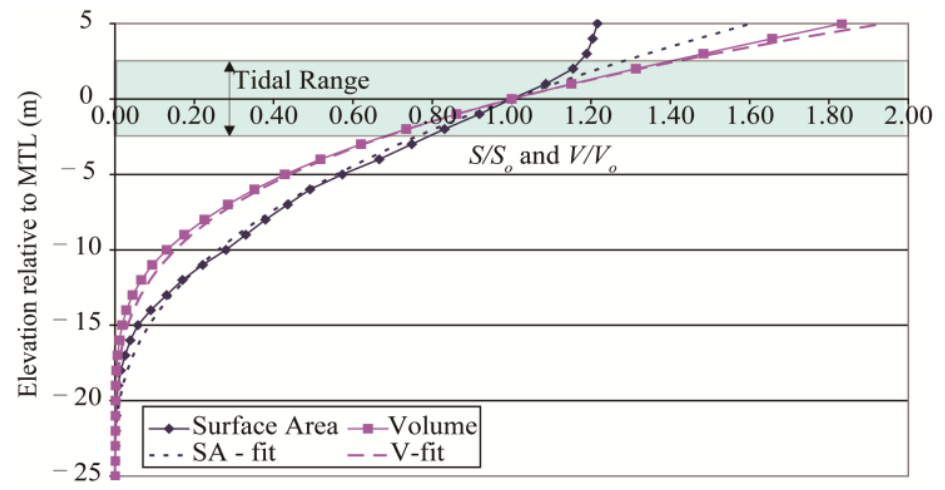

Fig. 3. Hypsometry of the Humber estuary compared to the power law functions for volume and surface area.

The maximum depth, $d$, and the shape parameter, $\alpha$, are constrained by Eq. (34), so that if one is known, the other can be estimated. Alternatively, they can be obtained as fitting parameters based on the existing bathymetry. Thereafter it is assumed that the shape parameter remains constant. The historic data for the last 70 years on the Humber estuary suggest that this is a reasonable assumption. When used in ASMITA, Eqs. (32)-(35) provide high and low water values based on updated values of the tidal prism, from which channel and flat volumes and surface areas are obtained.

\subsubsection{Relationships for Tidal Deltas}

The original development of ASMITA (and its application to the Wadden Sea) relied on the work of Walton and Adams (1976) to parameterise the equilibrium relationship for the tidal delta. This provides an empirical relationship for the volume of sediment in the delta over the surrounding bed level as, by relating the volume to the tidal prism: $V=a P^{b}$, where $V$ is the sediment volume, $P$ is the tidal prism, and $a$ and $b$ were defined as $6.57 \mathrm{E}-3$ and 1.23 , respectively, although the coefficients are usually adjusted when applied to specific inlets.

Subsequent work has explored the offshore extent of ebb-tidal deltas (Carr and Kraus, 2001; de Vriend et al., 1994) and the distance alongshore (Carr and Kraus, 2001; Hoan et al., 2011). However, these studies largely focused on micro and meso-tidal inlets. In contrast, estuaries and rias that are meso- or macro-tidal have very different morphologies and there is little knowledge to guide the determination of suitable equilibrium.

The plan area of the delta is estimated based on the maximum distance offshore and the up and down drift distances to the shore connection points, as defined in the literature. This could be defined 
as the area of semi-circle or a triangle and, for simplicity, the latter is adopted. Hence the plan area is given by: $S=0.5 L\left(W_{\mathrm{u}}+W_{\mathrm{d}}\right)$, where $L$ is the distance offshore, $W_{\mathrm{u}}$ is the updrift distance, $W_{\mathrm{d}}$ is the downdrift distance. These three variables have all been related to tidal prism and various workers (Carr and Kraus, 2001; de Vriend et al., 1994; Sha and van den Berg, 1993) have suggested various empirical relationships based on exposure and the number of jetties at the inlet mouth.

Although Hoan et al. (2011) modify the up and down drift relationships to account for the angle of prevailing wave attack and associated asymmetry of the delta, this is not included, given the overall uncertainty associated with the empirical relationships. Hence, relationships for exposed coast without jetties are given as:

$$
L=0.25 P^{0.4} ; \quad W_{\mathrm{u}}=0.09 P^{0.54} ; \quad W_{\mathrm{d}}=0.048 P^{0.57}
$$

Whence the plan area of the delta is given by: $S=\left(0.01125+0.006 P^{0.03}\right) P^{0.94}$.

\subsection{Equivalence with Regime Theory}

The regime method uses the following relationships to express the variation between key form parameters and the tidal discharge (Langbein, 1963; Myrick and Leopold, 1963):

$$
W \propto Q^{p} ; H \propto Q^{q} ; \quad A \propto Q^{r},
$$

where $r=p+q, W$ is the width, $H$ the hydraulic depth, $A$, the cross-sectional area, $Q$, the discharge, and $p, q$, and $r$ are exponents. In the literature these values are taken at mean tide level, or the level of peak discharge. The latter is generally considered more representative and is used here.

In contrast, ASMITA is based on an assumption that the morphology will adjust so that concentrations equal an equilibrium value, as governed by Eq. (2). This is implemented within the ASMITA model as a function of horizontal and vertical exchange rates, Eqs. (3) and (4) and an expression for the equilibrium volume, Eq. (1). The latter can be represented in a number of ways but the most widely used is to relate the volume to the tidal prism, i.e. $V_{\mathrm{e}}=\alpha P$, where $P$ is the tidal prism. Given that for a symmetric tide, $Q \approx \pi P / T_{\mathrm{p}}$, it is clear that $V_{\mathrm{e}}$ could be written in terms of discharge.

More generally, this is written with the prism to some power, $m$, following the initial observation of this relationship by O'Brien (1931). A number of authors have subsequently explored the physical basis of this equation (Friedrichs, 1995; Hughes, 2002; Kraus, 1998). Whereas Kraus and Hughes conclude that the prism or discharge is to some exponent. Friedrichs suggests that, for the case of a symmetric tide, the exponent is one. However, he then explains the variations about a value of one based on the role of tidal asymmetry, river flows and the development of a turbidity maxima. His resulting equation has the form:

$$
A \cdot H^{1 / 6}=N \cdot \sqrt{\frac{\rho \cdot g}{\tau_{s}}} \cdot Q^{\beta},
$$

where $N$ is Manning's friction coefficient, $\rho$ is the density of water, $g$ the acceleration due to gravity, $\tau_{\mathrm{s}}$ the total shear stress that is just stable and $\beta$ is the exponent that varies about one. A seaward decrease in shear stress should cause $\beta>1$ and be associated with flood dominant discharge, whereas a decrease of the shear stress in a landward direction should give $\beta<1$ and reflect ebb dominance. Friedrich 
reports this to be the case for the Thames and the Usk estuaries in the UK.

Now the along estuary variation of cross-section for a convergent estuary can be expressed as an exponential of the form $A=A_{\mathrm{o}} \exp \left(-x / L_{A}\right)$, where $A_{\mathrm{o}}$ is the area at the mouth and $L_{A}$ is the e-folding length of the cross-sectional area. It then follows that the volume to the same reference (MTL or level of peak discharge) is given by:

$$
V=\int_{0}^{L_{x}} A \mathrm{~d} x=A_{\mathrm{o}} \cdot L_{A}\left[1-\exp \left(\frac{-L_{x}}{L_{A}}\right)\right] \approx A_{\mathrm{o}} \cdot L_{A} .
$$

Combining Eqs. (38) and (39) the volume can also be written as a regime relationship, similar to those in equation (37), i.e. $V \propto Q^{s}$, and will vary in a similar manner, assuming that $L_{A}$ remains approximately constant when the system is perturbed. Hence, for typical coastal plain estuaries there should be an equivalence between a Regime model and ASMITA when using tidal prism as a basis for equilibrium. However, along channel variations and the recognition of the role of tidal asymmetry, river flows and the associated turbidity maxima, as characterised by the exponent, $\beta$, may need to be carefully examined if a one-to-one correspondence is to be established.

\subsection{Morphological Response Time}

Linearising Eq. (5) around the equilibrium, Kragtwijk et al. (2004) show that the morphological time scale can be expressed as:

$$
\tau=\frac{1}{c_{\mathrm{E}} \cdot n}\left(\frac{V_{\mathrm{e}}}{w_{\mathrm{s}} \cdot S}+\frac{V_{\mathrm{e}}}{\delta}\right) .
$$

This makes clear that the morphological e-folding time scale (or half-life) is a function of the system properties but not the disturbance. This equation is derived for a single element model and so also provides a response time scale for the system as a whole. For multiple elements, similar relationships can be derived for the individual elements (see Kragtwijk et al. (2004) for further details). When seeking to apply the model in some forms of hindcast against historical data, it is important to recognise that the changes, such as sea-level rise, or non-equilibrium advective flows (flows for which equilibrium is not defined by the global equilibrium concentration, $c_{\mathrm{E}}$ ) invoke a perturbation and hence a change in volumes in accordance with the equilibrium relationships adopted. For example, when including sea-level rise, it may be necessary to include an initial "spin-up" period to allow the model to adjust to the prevailing rate of rise. Subsequent changes are then correctly reproduced as perturbations on the initial rate. Similarly, if river inputs, or littoral drift are included there may be a need to include an adjustment to the rate of change equation, as explained below for advective flows below and in Part 2 for littoral drift.

\subsection{Advective Flows}

By default, the ASMITA model assumes that the equilibrium of the elements within the model domain are conditioned by the concentration in the "outside world", or external environment. When considering a delta/channel/flat system, as in the original applications, this global equilibrium is determined by the concentration in the "external" marine environment (i.e. a single value). The condition for equilibrium then requires that the volume rate of change goes to zero, Eq. (3), which is 
equivalent to zero gradient in the concentration field. If other sources are introduced, such as a river input, this remains the basis for equilibrium provided the concentration is the same as the global concentration. However, if the concentration associated with the other sources is not the same then Eq. (3) no longer represents an equilibrium state, or put another way, the additional source constitutes a perturbation to the system. One way of overcoming this is to determine the equilibrium taking account of the additional inputs, on the assumption that these are intrinsic to the state of equilibrium represented by the initial state of the system being modelled. This is predicated on the idea that the form of the estuary (bathymetry etc.) used to describe the existing state will be close to an equilibrium under the prevailing conditions and, consequently, the various inputs must all be represented in this state.

For a single element with the inclusion of a river input, Eq. (3) is modified as follows:

$$
\frac{\mathrm{d} V}{\mathrm{~d} t}=C\left[\left(q_{\mathrm{o}}+\delta_{\mathrm{e}}\right)\left(\frac{V_{\mathrm{e}}}{V}\right)^{n}-\left(q_{\mathrm{r}}+\delta_{\mathrm{e}}\right)\right],
$$

where $q_{\mathrm{o}}$ is discharge to the marine environment, $q_{\mathrm{r}}$ is the river discharge and $\delta_{\mathrm{e}}$ is the horizontal exchange rate. In order to have a different concentration associated with the river flow but allow $c_{\mathrm{E}}$ to be factored into the coefficient $C$ in Eq. (41) the imported concentrations are expressed as $c_{\mathrm{i}}=k_{\mathrm{i}} c_{\mathrm{E}}$. With some re-arrangement it can be shown that $\mathrm{d} V / \mathrm{d} t=>0$ if the equilibrium volume is factored by:

$$
\alpha=\left(\frac{k \cdot q_{\mathrm{r}}+\delta_{\mathrm{E}}}{q_{\mathrm{o}}+\delta_{\mathrm{E}}}\right)^{1 / n} \text { so that: } \frac{\mathrm{d} V}{\mathrm{~d} t}=C\left[\left(q_{\mathrm{o}}+\delta_{\mathrm{e}}\right)\left(\frac{\alpha \cdot V_{\mathrm{e}}}{V}\right)^{n}-\right]\left(q_{\mathrm{r}}+\delta_{\mathrm{e}}\right) .
$$

In a single element model, the flow in and out must balance to ensure mass continuity, so that $q_{\mathrm{o}}$ $=q_{\mathrm{r}}$. However, they are kept explicit here to illustrate the basis of the implementation in matrix form for multiple elements, which is detailed in the Appendix.

\section{Typical Application}

To illustrate the application of the model based on its original formulation we make use of a twoelement model, comprising a channel and a tidal flat, with dimensions and parameters that are based on the Humber estuary (Townend et al., 2008). The Humber Estuary is located on the North Sea coast of England and drains a catchment area of just under $24000 \mathrm{~km}^{2}$ with an annual average discharge of some $250 \mathrm{~m}^{3} / \mathrm{s}$. The estuary is macro-tidal, with a mean tidal range of $6 \mathrm{~m}$ and extends some $145 \mathrm{~km}$ to the tidal limit. Suspended sediment loads are high with a turbidity maximum that moves between Hull, $30 \mathrm{~km}$ from the mouth, and Selby, $95 \mathrm{~km}$ from the mouth, depending on the seasonal conditions. The variations in the geomorphology from the mouth to the tidal limit mean that this estuary could be schematised into elements in many different ways (Rossington et al., 2007). The results from 3 different model runs using the 2 element model (channel and tidal flat) are shown in Fig. 4. The left hand plot is the tidal channel and the right hand plot is the tidal flat. Each plot shows how the volume (moving, equilibrium and fixed; see Eq. (6)) changes with time. The three cases are:

(i) sea-level rise of $1.8 \mathrm{~mm} / \mathrm{a}$ - indicated by the linear lines;

(ii) sea-level rise and a cyclic variation in tidal range based on the lunar nodal tidal signal; 
(iii) sea-level rise with an advective (river) flow but no account taken of this in the equilibrium condition; see Eqs. (42) and (43). This is only shown for the moving surface. Results for the other two volumes have a similar offset.

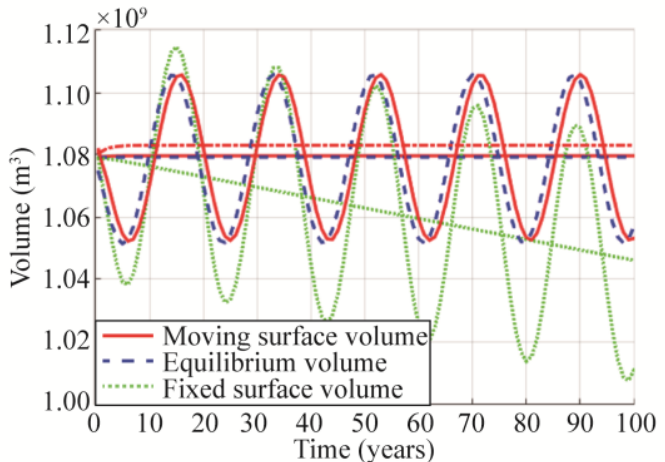

(a) Channel

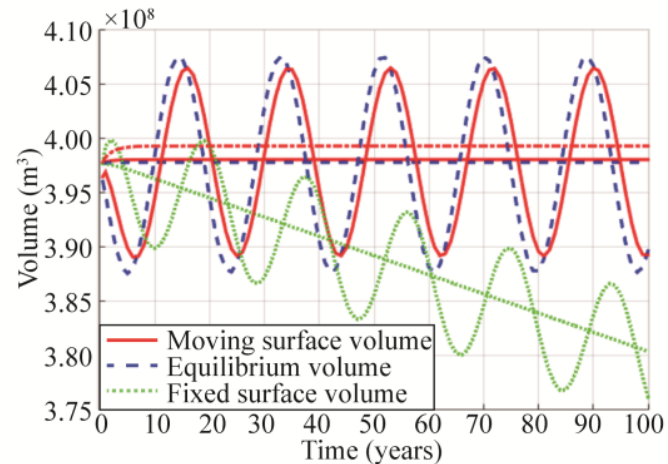

(b) Tidal Flat

Fig. 4. Illustration of (wet) volume changes for a 2 element model. Volumes are moving surface, equilibrium and fixed surface for a sea-level rise of $1.8 \mathrm{~mm} / \mathrm{a}$ (linear change) and sea-level rise plus the 18.6 year lunar nodal cycle. The additional moving surface line (dash-dot) includes a river flow which is not accounted for in the equilibrium condition.

It is also relatively easy to impose changes, to reflect dredging operations, reclamations or managed realignments, by introducing the change $(\Delta V(t)$ and $\Delta S(t))$ into Eq. (6) for the fixed and moving surface volumes. For a system that is initially near to equilibrium and not subject to other changes, such as sea-level rise, the response to a change at some time, $t$, is to restore the initial state over a time scale that reflects the morphological time-scale of the system (Jeuken et al., 2003).

The model is also very efficient and hence well suited to very long simulations or use in uncertainty analysis where ensemble or Monte Carlo techniques are being used. This is nicely illustrated by the application of the model to the Humber estuary in the UK to consider changes over Holocene (2000 years) and Historical (150 years) time scales. This was originally investigated using an empirical model (Townend et al., 2007) but a similar result is readily obtained using ASMITA, Fig. 5.

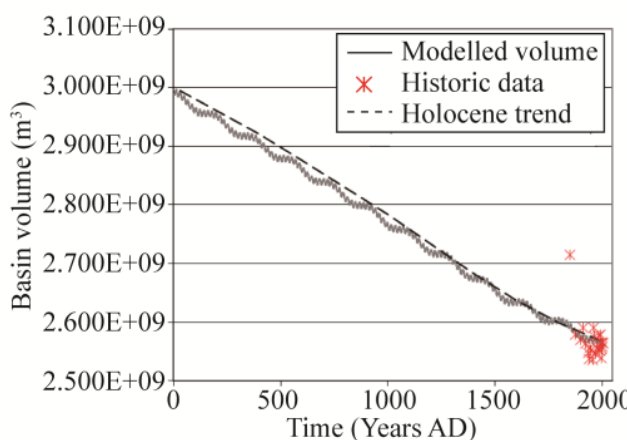

(a) Holocene

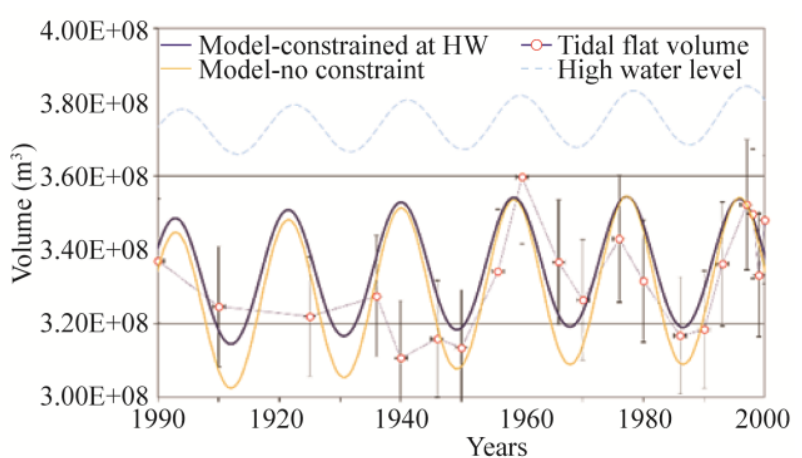

(b) Historical

Fig. 5. Comparison of modelled estuary volume with (a) estimates of the rate of basin infilling derived from the stratigraphic records for the Humber Estuary (Rees et al., 2000) and (b) historical data derived from bathymetric charts. Measured data includes error bars $( \pm 1$ year and $\pm 5 \%$ volume or area).

\section{Conclusions}


The ASMITA model has been under continuous development over the last two decades since it was first proposed in 1998. As an aggregated type of model based on prescribed equilibrium relationships, it aims to explore the bulk morphodynamic changes (in terms of volume change) of large-scale estuarine and coastal systems over relatively long term, in contrast to the fast-scale processbased models (e.g., Delft3D) which intend to capture the details of bed level change. Because of its simplicity and effectiveness, the ASMITA model has been used in a number of applications, showing its competence in, e.g., assessing the morphodynamic response of tidal inlets and estuaries to sea-level rise and anthropogenic interventions. During the last decade, a number of extensions to explore other inlet and estuary dynamics have been developed within the mass-balance framework of ASMITA. These are presented in Part 2 and include (1) tidal pumping, (2) saltmarsh and storage element, (3) transport of mixed sediment, (4) variable area and constraints, (5) literal drift for delta and (6) the effect of waves. Adding more complexity, these extensions widen the application possibilities of the model and make the model more realistic, while keep the advantage of obeying the prescribed morphological equilibrium over the long-term. Therefore, the ASMITA-type of models represent a different modelling philosophy, which can complement process-based models, especially when dealing with large-scale and long-term simulations.

Acknowledgements - Many colleagues have contributed to the developments reported in this paper. Contributions by Tjerk Zitman at TU Delft, Adrian Wright at ABPmer, Kate Rossington, Michiel Knaapen, and Jeremy Speerman at HR Wallingford, Davide Bonaldo at CNR-ISMAR are duly acknowledged.

\section{References}

Cao, S. and Knight, D., 1997. Entropy-based design approach of threshold alluvial channels, J. Hydraul. Res., 35(4): 505-524.

Carr, E. E. and Kraus, N. C., 2001. Morphologic Asymmetries at Entrances to Tidal Inlets, CHETN-IV-33, U.S. Army Engineer Research and Development Center, Vicksburg, MS.

Dal Monte, L. and Di Silvio, G., 2004. Sediment concentration in tidal lagoons. A contribution to long-term morphological modelling, J. Marine Syst., 51(1-4): 243-255.

de Vriend, H. J., Bakker, W. T. and Bilse, D. P., 1994. A morphological behaviour model for the outer delta of mixed-energy tidal inlets, Coast. Eng., 23(3-4): 305-327.

Di Silvio, G., 1989. Modelling the morphological evolution of tidal lagoons and their equilibrium configurations, IAHR Congress, IAHR, pp. C-169-C-175.

Di Silvio, G., Dall' Angelo, C., Bonaldo, D. and Fasolato, G., 2010. Long-term model of planimetric and bathymetric evolution of a tidal lagoon, Cont. Shelf Res., 30(8): 894-903.

Dissanayake, D. M. P. K., Ranasinghe, R., Roelvink, J. A. and Wang, Z. B., 2011. Process-based and semiempirical modelling approaches on tidal inlet evolution, J. Coastal Res., (SI64): 1013-1017.

Dronkers, J., 1978. Longitudinal dispersion in shallow well-mixed estuaries, in: B. L. Edge (Ed.), International Conference on Coastal Engineering, ASCE, 2761-2777.

Dronkers, J., 2005. Dynamics of coastal systems, Advanced Series in Ocean Engineering, 25, World Scientific, Singapore.

Eysink, W. D., 1990. Morphologic response of tidal basins to changes, Proceeding of the 22nd International Conference on Coastal Engineering, Delft, The Netherlands, 1948-1961.

Eysink, W. D., 1991. Simple morphologic relationships for estuaries and tidal channels - handy tools for 
engineering, Proceedings of the International Conference on Coastal and Port Engineering in Developing Countries, 1003-1013.

Eysink, W. D. and Biegel, E. J., 1992. Impact of sea level rise on the morphology of the Wadden Sea in the scope of its ecological function, Investigations on Empirical Morphological Relations, Report H1300, Phase 3, Delft Hydraulics.

Friedrichs, C. T., 1995. Stability shear stress and equilibrium cross-sectional geometry of sheltered tidal channels, J. Coastal Res., 11(4): 1062-1074.

Friedrichs, C. T. and Aubrey, D. G., 1996. Uniform bottom shear stress and equilibrium hypsometry of intertidal flats, in: C. Pattiaratchi (Ed.), Mixing in Estuaries and Coastal Seas, Coastal and Estuarine Studies, American Geophysical Union, Washington, 405-429.

Gallappatti, G. and Vreugdenhil, C. B., 1985. A depth integrated model for suspended sediment transport, $J$. Hydraul. Res., 23(4): 359-375.

Hoan, L. X., Hanson, H., Larson, M., Kato, S. and Aoki, S., 2011. Modeling Regional Sediment Transport and Barrier Elongation on Long Island Coast, United States, 2011 Conference on Coastal Engineering Practice: Engineering Sustainable Coastal Development, San Diego, California, 473-486.

Hughes, S. A., 2002. Equilibrium cross sectional area at tidal inlets, J. Coastal Res., 18(1): 160-174.

Jarrett, J. T., 1976. Tidal Prism-Inlet Area Relationship, Report 3.

Jeuken, M. C. J. L., Wang, Z. B., Keiller, D., Townend, I. H. and Liek, G. A., 2003. Morphological response of estuaries to nodal tide variation, International Conference on Estuaries and Coasts (ICEC-2003), Hangzhou, China, 167-173.

Kragtwijk, N. G., Stive, M. J. F., Wang, Z. B. and Zitman, T. J., 2004. Morphological response of tidal basins to human interventions, Coast. Eng., 51, 207-221.

Kraus, N. C., 1998. Inlet cross-sectional area calculated by process-based model, Proceedings of the 26th International Conference on Coastal Engineering, Copenhagen, Denmark, 3265-3278.

Langbein, W. B., 1963. The hydraulic geometry of a shallow estuary, Bulletin-International Association of Scientific Hydrology, 8(3): 84-94.

Lewis, R. E., 1997. Dispersion in Estuaries and Coastal Waters, John Wiley \& Sons, Chichester, England.

Myrick, R. M. and Leopold, L. B., 1963. Hydraulic Geometry of A Small Tidal Estuary, 422-B, Washington.

O’ Brien, M. P., 1931. Estuary tidal prism related to entrance areas, Civil Eng., 1(8): 738-739.

Rees, J. G., Ridgway, J., Ellis, S., Knox, R. W. O., Newsham, R. and Parkes, A., 2000. Holocene sediment storage in the Humber Estuary, in: I. Shennan, J. E. Andrews (Eds.), Holocene Land-Ocean Interaction and Environmental Change Around the North Sea, Special Publications 166, Geological Society, London, $119-143$.

Renger, E. and Partenscky, H. W., 1974. Stability criteria for tidal basins, Proceedings of the 14th International Conference on Coastal Engineering, Copenhagen, Denmark, 1605-1618.

Rossington, K., Nicholls, R. J., Knaapen, M. A. F. and Wang, Z. B., 2007. Morphological Behaviour of UK Estuaries under Conditions of Accelerating Sea Level Rise, in: C.M. Dohmen-Janssen, S.J.M.H. Hulscher (Eds.), RCEM2007. River, Coastal and Estuarine Morphodynamics, Taylor \& Francis.

Savenije, H. H. G., 2005. Salinity and Tides in Alluvial Estuaries, Elsevier, Amsterdam, Netherlands.

Sha, L. P. and van den Berg, J. H., 1993. Variations in the ebb-tidal delta geometry along the coast of The Netherlands and the German Bight, J. Coastal Res., 9(3): 730-746.

Townend, I. H., 2005. An examination of empirical stability relationships for UK estuaries, J. Coastal Res., 21(5): $1042-1053$. 
Townend, I. H., 2010. An exploration of equilibrium in Venice Lagoon using an idealised form model, Cont. Shelf Res., 30(8): 984-999.

Townend, I. H., 2012. The estimation of estuary dimensions using a simplified form model and the exogenous controls, Earth Surf. Proc. Land., 37, 1573-1583.

Townend, I. H., Wang, Z. B. and Rees, J. G., 2007. Millennial to annual volume changes in the Humber Estuary, Proc. R. Soc. A, 463, 837-854.

Townend, I. H., Wang, Z. B., Spearman, J. and Wright, A. D., 2008. Volume and surface area changes in estuaries and tidal inlets, Proceedings of the 31st International Conference on Coastal Engineering, Hamburg, Germany, 4495-4507.

van Goor, M. A., Zitman, T. J., Wang, Z. B. and Stive, M. J. F., 2003. Impact of sea-level rise on the morphological equilibrium state of tidal inlets, Mar. Geol., 202(3-4): 211-227.

Walton, T. D. and Adams, W. D., 1976. Capacity of inlet outer bars to store sand, Proceedings of the 15th International Conference on Coastal Engineering, Honolulu, Hawaii, 1919-1936.

Wang, Z. B., 1992. Theoretical analysis on depth-integrated modelling of suspended sediment transport, $J$. Hydraul. Res., 30(3): 403-420.

Wang, Z. B., de Vriend, H. J., Stive, M. J. F. and Townend, I. H., 2008. On the parameter setting of semi-empirical long-term morphological models for estuaries and tidal lagoons, in: C.M. Dohmen-Janssen, S.J.M.H. Hulscher (Eds.), River, Coastal and Estuarine Morphodynamics, Taylor \& Francis, 103-111.

Wang, Z. B., Karssen, B., Fokkink, R. J. and Langerak, A., 1998. A dynamic-empirical model for estuarine morphology, in: J. Dronkers, M.B.A.M. Scheffers (Eds.), Physics of Estuaries and Coastal Seas, Balkema, Rotterdam, 279-286.

Wang, Z. B. and Townend, I. H., 2012. Influence of the nodal tide on the morphological response of estuaries, Mar. Geol., 291-294, 73-82.

\section{Appendix}

The equations presented for the single element model can be conveniently written in matrix form to represent a multi-element model. Here we follow the naming convention of Kragtwijk et al. (2004) for volumes and add some additional terms to make the model more general with respect to dispersion, advection and the sources of perturbations in the volume.

For a multi-element model, the variables used in this Appendix are defined as either vectors or matrices, as follows:

$\underline{V}: \quad$ element volumes;

$\underline{S}$ : element surface areas;

$c_{\mathrm{e}}: \quad$ local equilibrium concentrations;

$\underline{c}: \quad$ element concentrations;

concentrations for fluxes into the system

$\underline{c_{\text {ext }}}:$ from the environment;

\footnotetext{
advective flows into the system from the

$\underline{q_{\mathrm{ext}}}: \quad$ environment
}

$\underline{\gamma}: \quad$ volume ratios $\left(V_{\mathrm{ke}} / V_{\mathrm{k}}\right)^{n}$;

$\underline{\sigma}: \quad$ surface area ratios $\left(S_{\mathrm{ke}} / S_{\mathrm{k}}\right)^{\mathrm{abs}(n)}$;

$\delta_{\text {ext }}:$ horizontal exchange coefficients with environment; concentration transport exponent.

$\underline{n}$ : $\quad$ positive for wet volumes and negative for sediment volumes;

$\underline{c_{\mathrm{b}}}: \quad$ concentration of bed; 
Diagonal matrices:

D: horizontal exchange between elements

$\boldsymbol{W}: \quad$ vertical exchange coefficient $w$

$\boldsymbol{Q}$ : advective exchange between elements

$S: \quad$ surface area

$\boldsymbol{B}$ : expression to scale rate of change

I: $\quad$ unit or identity matrix

$\underline{d}$ : expression for offset to rate of change

$\boldsymbol{M}$ : unit matrix with sign of $n$

The matrix $\boldsymbol{D}$ reflects the structure of the horizontal exchange between elements and with the outside world. For an $n$-element system with all elements linked and exchanging sediment with external environments, this would take the following form:

$$
\boldsymbol{D}=\left(\begin{array}{cccc}
\sum \delta_{1, n}+\delta_{1, E} & -\delta_{1,2} & \cdots & -\delta_{1, n} \\
-\delta_{2,1} & \sum \delta_{2, n}+\delta_{2, E} & \cdots & -\delta_{2, n} \\
\cdots & \cdots & \cdots & \cdots \\
-\delta_{n, 1} & -\delta_{n, 2} & \cdots & \sum \delta_{n, n}+\delta_{n, E}
\end{array}\right)
$$

For dispersive horizontal exchange the matrix is symmetric because $\delta_{i, j}=\delta_{j, i}$ and the direction of transport depends on the concentration gradient. However, for advective transport, the flow has a specified direction and we adopt the convention of transport from element $i$ to element $j$. The matrix for an n-element system is then given by:

$$
\boldsymbol{Q}=\left(\begin{array}{cccc}
\sum q_{1, n}+q_{1, E} & -q_{1,2} & \ldots & -q_{1, n} \\
-q_{2,1} & \sum q_{2, n}+q_{2, E} & \cdots & -q_{2, n} \\
\cdots & \cdots & \cdots & \cdots \\
-q_{n, 1} & -q_{n, 2} & \cdots & \sum q_{n, n}+q_{n, E}
\end{array}\right),
$$

where $q_{n, E}$ all relate to fluxes out of the system. In order to ensure continuity of water mass we also require that, for each element, the sum of the discharges in and out of the element is zero, ie $\sum q_{i}=0$. However, provision is made for the sediment concentrations associated with these discharges to vary. The basic equations can now be written:

$$
\begin{aligned}
& \frac{\mathrm{d} \underline{V}}{\mathrm{~d} t}=\frac{1}{c_{\mathrm{b}}} \boldsymbol{M} \boldsymbol{W} \boldsymbol{S}\left(\underline{c_{\mathrm{e}}}-\underline{c}\right) ; \\
& \left(\boldsymbol{D}+\boldsymbol{Q}^{\mathrm{T}}\right) \underline{c}=\boldsymbol{W} \boldsymbol{S}\left(\underline{c_{\mathrm{e}}}-\underline{c}\right)+c_{\mathrm{E}}\left(\underline{\delta_{\text {ext }}}+\underline{k_{c} \cdot q_{\mathrm{ext}}}\right) ; \\
& \underline{c_{\mathrm{e}}}=c_{\mathrm{E}} \underline{\gamma} \text { and } \underline{k_{c}}=\underline{c_{\text {ext }}} / c_{\mathrm{E}},
\end{aligned}
$$

The basis for calculating the change in volume due to some perturbation is then as follows:

$$
\frac{\mathrm{d} V}{\mathrm{~d} t}=\frac{1}{\underline{c_{\mathrm{b}}}}(\boldsymbol{B} \underline{\gamma}-\underline{d})+\underline{\Delta V}
$$

where 


$$
\begin{aligned}
& \boldsymbol{B}=c_{\mathrm{E}} \boldsymbol{M W S}\left[\boldsymbol{I}-\left(\boldsymbol{D}+\mathbf{Q}^{\mathrm{T}}+\boldsymbol{W S}\right)^{-1} \boldsymbol{W S}\right] ; \\
& \underline{d}=c_{\mathrm{E}} \boldsymbol{M W S}\left(\boldsymbol{D}+\boldsymbol{Q}^{\mathrm{T}}+\boldsymbol{W S}\right)^{-1}\left(\underline{\delta_{\mathrm{ext}}}+\underline{k_{c}} \cdot \underline{q_{\mathrm{ext}}}\right) .
\end{aligned}
$$

The term $\underline{\Delta V}$ refers to any other changes in volume, introduced at any given time, within each element, eg due to reclamation or dredging. The change determined by Eq. (A.4) is the morphological adjustment relative to the initial volume and so does not include changes in water levels and tidal range but the volumes used in defining $\underline{\gamma}$ are based on the total water volume of the element at any given time interval. As explained in the main text, these are referred to as $V_{\mathrm{f}}$ and $V_{\mathrm{m}}$, respectively. When using the ratio of element volume to tidal prism as the basis for equilibrium, the equilibrium, fixed and moving volumes are given by:

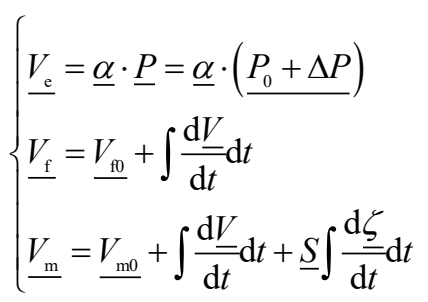

Stability requires the numerical propagation to be faster than the physical speed of propagation of any given property. In terms of advection this requires that the volume cannot infill in a single time step, so that $q \cdot c \cdot \mathrm{d} t / V \leq 1$. A similar requirement can be imposed for dispersion, with a dispersion coefficient $K: K \cdot \mathrm{d} t /\left(2 X^{2}\right) \leq 1$, where $X$ is some appropriate excursion length. In ASMITA we are essentially dealing with horizontal fluxes $\boldsymbol{Q}$ and $\boldsymbol{D}$ (both $\mathrm{m}^{3} / \mathrm{s}$ ). We can therefore write the stability criteria as:

$$
\Delta t \leq \min \left[\frac{\boldsymbol{V}}{(\boldsymbol{Q}+\boldsymbol{D}) \cdot c}\right] .
$$

\section{Advective Flow Equilibrium}

If an advective flow, such as a river flow, is included, this implies a perturbation to the system unless accounted for in the rate of change equations, such as Eq. (P1.3). This is addressed by taking account of the additional inputs, on the assumption that these are intrinsic to the state of equilibrium represented by the initial state of the system being modelled. To account for this, the equilibrium volume and area are corrected using the arguments that lead to Eqs. (32) and (33) for a single element. In matrix form this adjustment, $\alpha$, can be obtained from the initial conditions, as follows:

$$
\underline{S_{\mathrm{e}}}=\underline{S_{\mathrm{e} 0}}\left(\boldsymbol{F}^{-1} \cdot \underline{e}\right)^{1 / n} \quad \text { and } \quad \underline{V_{\mathrm{e}}}=\underline{V_{\mathrm{e} 0}}\left(\boldsymbol{B}^{-1} \cdot \underline{d}\right)^{1 / n} .
$$

